\title{
BEAM DUMP WINDOW DESIGN FOR THE SPALLATION NEUTRON SOURCE*
}

G. Murdoch, A. Decarlo, S. Henderson, S. Kim, K Potter, T. Roseberry, Oak Ridge National Laboratory, USA J. Rank, D. Raparia, Brookhaven National Laboratory, USA

\section{Abstract}

The Spallation Neutron Source accelerator systems will provide a $1 \mathrm{GeV}, 1.44 \mathrm{MW}$ proton beam to a liquid mercury target for neutron production. Beam tuning dumps are provided at the end of the linac (the Linac Dump) and in the Ring-to-Target transport line (the Extraction Dump) [1]. Thin windows are required to separate the accelerator vacuum from the poor vacuum upstream of the beam dump. There are several challenging engineering issues that have been addressed in the window design. Namely, handling of the high local power density deposited by the stripped electrons from the $\mathrm{H}$ - beam accelerated in the linac, and the need for lowexposure removal and replacement of an activated window. The thermal design of the linac dump window is presented, as is the design of a vacuum clamp and mechanism that allows remote removal and replacement of the window.

\section{INTRODUCTION}

The SNS linac zero degree beam dump is to be passively cooled dissipating $7.5 \mathrm{~kW}$ of beam power. The beam flight tube immediately upstream of the dump will be either evacuated or back filled with helium, to separate this medium from the accelerator machine vacuum a beam window is proposed.

\section{Physics}

\section{DESIGN PARAMETERS}

The window must be able to withstand continuous pulses of $2.1 \mathrm{e} 14 \mathrm{H}$ - particles/pulse with a pulse duration of $1 \mathrm{~ms}$ at a frequency of $0.2 \mathrm{~Hz}$; continuous running is simulated as the worst case scenario. The 2-D gaussian proton beam power density profile for Inconel with the above conditions is given in Figure 1 assuming a window thickness of $2 \mathrm{~mm}$ [2].

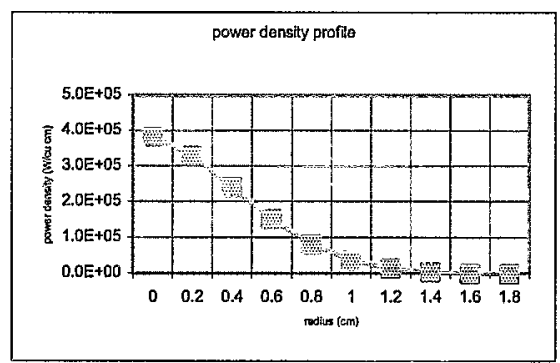

Figure 1

\section{Mechanical}

Several criteria must be considered during the mechanical design process. From an operations/maintenance view point a passive window design satisfying the physics parameters above would be the optimum solution i.e. no active cooling apart from convective cooling to air. Also, the window design must be integrated into the overall linac dump beam line design with consideration for subsequent handling issues such as shielding and remote removal and installation.

\section{MECHANICAL DESIGN}

A circular domed window $80 \mathrm{~mm}$ in diameter (beam diameter is $40 \mathrm{~mm}$ ) is adopted as the base design for thermal analysis. Initial runs indicated that the maximum thermal stress can be reduced by $\sim 40 \%$ if the beam impinges on the outer surface of the dome instead of the inner surface, it is likely this geometry creates less restriction to thermal expansion of the high temperature surface. Adopting a domed window is also advantageous from a structural integrity point of view. Three radial fins are added to promote convection. Three materials are considered, Aluminum 6061-T6, GlidCop Al-15 \& Inconel 718. It is intended to integrate the window design with two EVAC type vacuum flanges, this allows the window and flanges to be designed as an integral component. Also, to aid handling a remote vacuum clamp will be designed.

\section{THERMAL ANALYSIS}

The window is modeled as a 2-D axisymmetric running a transient thermal analysis to mimic the pulsed beam parameters. The beam loading is input to the ANSYS solution solver via a text program written to enable the specific beam conditions to be applied. Typical Inconel 718 values for the proton heat generation across a window thickness of $2 \mathrm{~mm}$ and electron heat generation (across the first $0.5 \mathrm{~mm}$ for Aluminum and $0.2 \mathrm{~mm}$ for GlidCop \& Inconel [3]) are given in Figure 2, these are derived from Figure 1 using the appropriate material deposition ratios [4]. The incremental values are applied to the model nodes as a function of radius from the center of the window outwards.

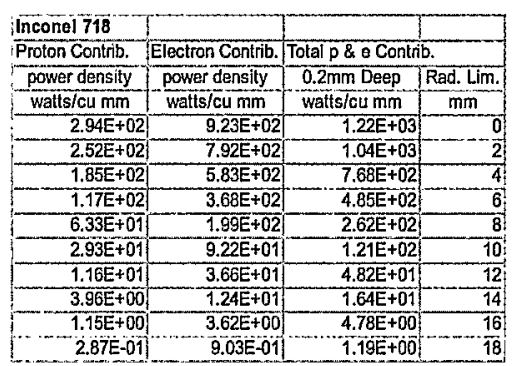

Figure 2

\section{ANALYSIS RESULTS}

Considering the results table of Figure 3, the maximum stress in Aluminum after $45.01 \mathrm{~s}$ is $158 \mathrm{~N} / \mathrm{mm}^{2}$, which is $80 \%$ of the yield strength of the material at $200 \mathrm{C}$. More the U.S. Department of Energy. 
importantly it is $\sim 3$ times the fatigue endurance limit. Running the window consistently above the fatigue limit would pose questions on potential crack propagation and consequently lifetime issues.

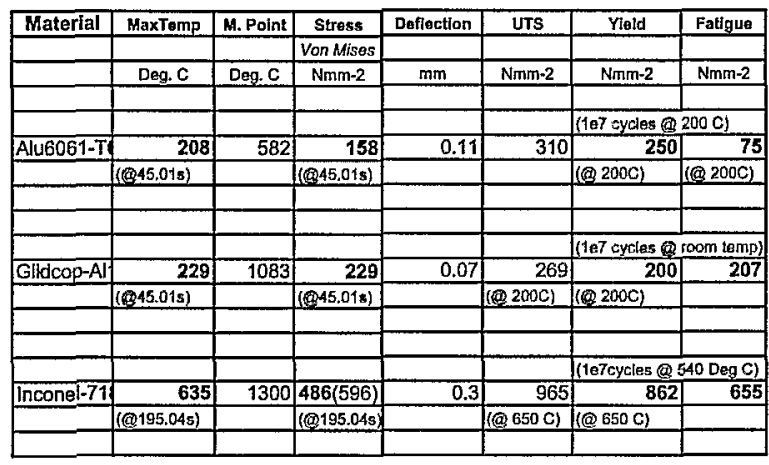

Figure 3

The maximum stress seen in the GlidCop after $45.01 \mathrm{~s}$ is $229 \mathrm{~N} / \mathrm{mm}^{2}$, which is above the yield strength of the material at $200 \mathrm{C}$. Comparison with fatigue values is difficult as data at elevated temperatures is scarce. Due to the poor diffusivity of Inconel the analysis is run for a longer period of time to achieve near equilibrium conditions, Figures 4 shows the transient analysis plot for Inconel-718. The maximum stress seen in the Inconel window is $596 \mathrm{~N} / \mathrm{mm}^{2}$, (after the first pulse) but tails off to a maximum value of $486 \mathrm{~N} / \mathrm{mm}^{2}$, at $195.04 \mathrm{~s}$, shown in Figure 5. This is probably due to the window absorbing heat, slowly warming and consequently causing a reduction in the temperature differential across the window. These stress values compare favorably with a yield strength of $862 \mathrm{~N} / \mathrm{mm}^{2}$, at $650 \mathrm{C}$ and a fatigue endurance limit of $655 \mathrm{~N} / \mathrm{mm}^{2}$, for $1 \mathrm{e} 7$ cycles at $540 \mathrm{C}$.

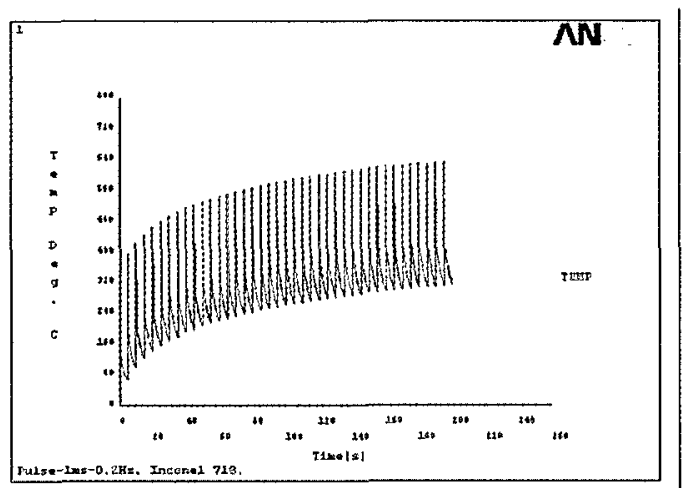

Figure 4

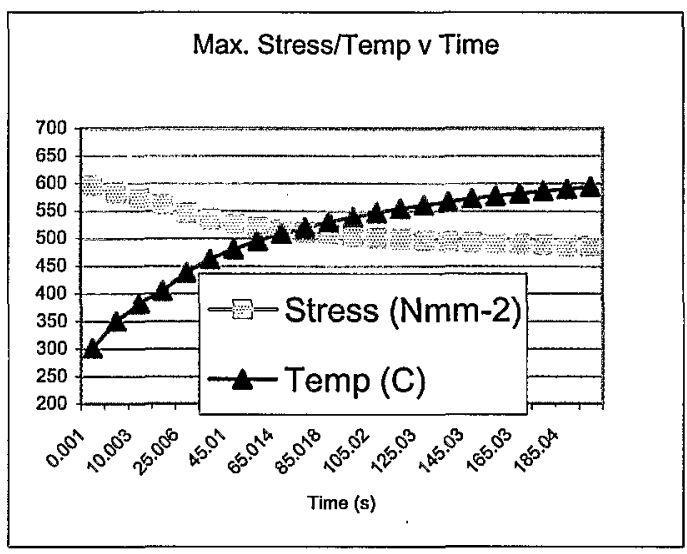

Figure 5

The stress and temperature fringe profiles for Inconel-718 are shown in Figure $6 \& 7$ respectively. Consideration of the principal stresses shows a maximum tensile stress value of $213 \mathrm{~N} / \mathrm{mm}^{2}$ and the maximum compressive stress value of $474 \mathrm{~N} / \mathrm{mm}^{2}$, this compressive stress is likely to inhibit crack propagation in the high temperature area.
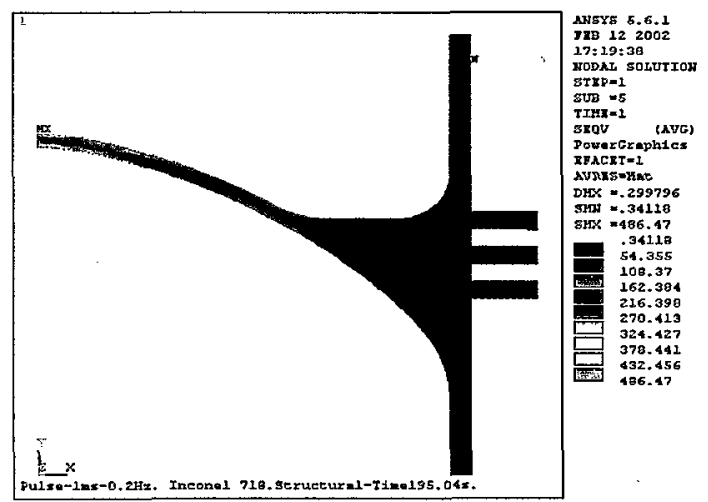

Figure 6

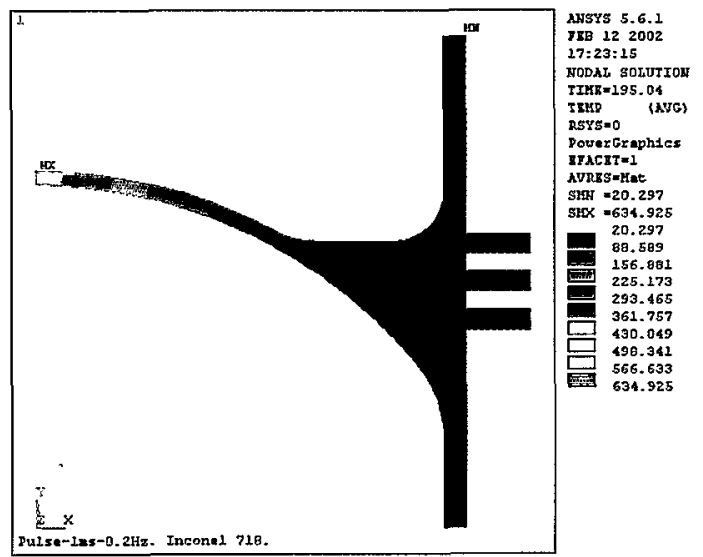

Figure 7 


\section{ANALYSIS CONCLUSIONS}

For a passively cooled window Inconel-718 is clearly the most robust choice of the three materials analyzed. One concern is continuous running at temperatures in the order of $635 \mathrm{C}$, although this is localized at beam center. Radiation heat transfer from the window surface has not been considered but basic calculations show that the radiated heat is negligible, being in the order of $1-5$ watts depending on the emissivity value chosen. The stress values are well within the yield and fatigue limits quoted in the extensive literature available for the material.

Inconel-718 is a recognized quantity from a proton irradiation lifetime point of view, however, it does contain longer half-life isotopes than either GlidCop or Aluminum and consequently will remain more activated. This will have to be taken into consideration in all work planning as an ALARA issue. The choice of a high integrity material such as Inconel-718 should lead to less down time and consequently improved machine availability. A 3-D ProE model of the finalized window design is shown in Figure 8.

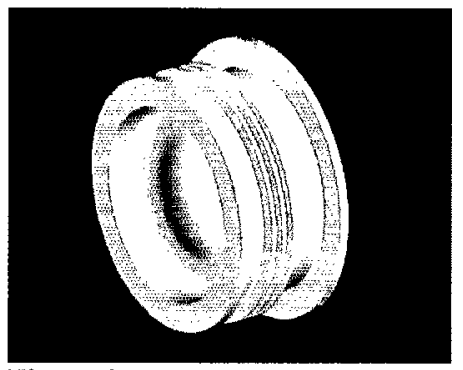

Figure 8

\section{WINDOW REPLACEMENT}

In the event of a window failure the window would have to be removed and replaced. It is likely that this could not be done by hands-on maintenance because of high residual radiation dose rates from the window material and surrounding equipment. Consequently, a window change scenario was addressed with many design options studied.

Two remote vacuum clamp designs have been pursued both of which are built and ready for testing. One version of the clamp is shown in Figure 9. The clamp is designed to utilize a standard EVAC type flange.

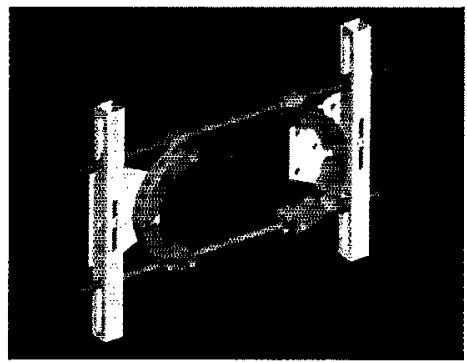

Figure 9
A test rig that will be used to validate the vacuum clamps and mimic a full window change scenario has also been designed and manufactured, this is shown in Figure 10.

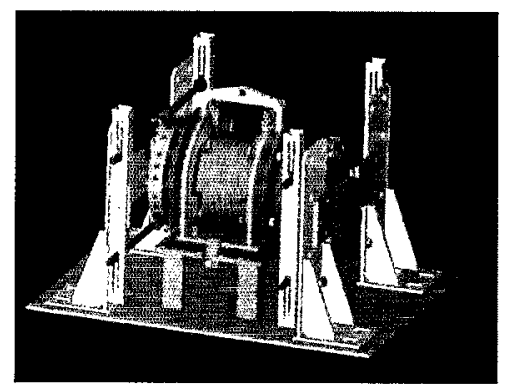

Figure 10

The test rig design is based on the handling concept that the window assembly shown in Figure 11 would be removed each time a window is replaced. A cradle will be lowered into position over the bellows assembly, the vacuum clamps are then opened and the cradle manipulated to compress the bellows. Once the bellows are compressed the window assembly including vacuum seals that are captive to the assembly can be removed. Provision has been made in the design for testing two proprietary vacuum seals, EVAC aluminum diamond and helicoflex delta.

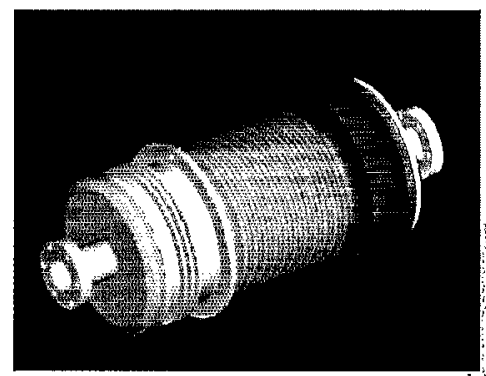

Figure 11 REFERENCES

[1] Deepak Raparia et al., Brookhaven National Laboratory, these proceedings.

[2] Deepak Raparia, Broôkhàven National Laboratory, USA Private Communication.

[3] Stuart Henderson, SNS Project, Oak Ridge National Laboratory, Private Communication, Studies in Penetration of Charged Particles in Matter, Nuclear Science Series, Report No. 39.

[4] Franz Gallmeier, SNS Project, Oak Ridge National Laboratory, Private Communication.

*SNS is a collaboration of six US National Laboratories: Argonne National Laboratory (ANL), Brookhaven National Laboratory (BNL), Thomas Jefferson National Accelerator Facility (TJNAF), Los Alamos National Laboratory (LANL), Lawrence Berkeley National Laboratory (LBNL), and Oak Ridge National Laboratory (ORNL). SNS is managed by UT-Battelle, LLC, under contract DE-AC05-00OR22725 for the U.S. Department of Energy. 